\title{
VELOCITY DISTRIBUTION OF UNSTEADY LAMINAR FLOW FOR VISCOUS FLUID
}

\author{
M. A. Haque \\ Department of Applied Mathematics,University of Rajshahi,Rajshahi-6205,Bangladesh
}

Received 11.08.08 Accepted 30.08.08

\begin{abstract}
In this paper an attempt has been made to investigate the velocity profile of unsteady laminar flow of incompressible viscous fluid. The method of separation of variable is used to determine the solutions of the governing differential equations. Time varying pressure gradient is considered for poiseuille flow. The velocity profiles for the various types of flow are shown by the figures.
\end{abstract}

\section{Introduction}

The steady and unsteady flow of viscous incompressible fluid between two parallel plates with constant pressure gradient have been presented in the standard books of Bachelor [1], Chorlton [2], Lamb [4], Landau \& Lifshitz [5], Milne Thomson [6], Pai [7], and others. In describing the unsteady flow of viscous fluid between two parallel plates Sengupta, Rahman \& Kandar [9] have considered time varying pressure gradients. In [9] Laplace transformation is used to determine the solutions of the differential equations. In this paper an attempt has been made to study the velocity profile of various types of unsteady two dimensional flow of incompressible viscous fluid between two parallel plates. In describing time varying pressure gradient for poiseuille flow some suitable functions are considered here. A special case is considered in this paper. Crank Nicholson method is used to determine the figures of the special case.

\section{Mathematical formulation}

Equation for conservation of mass:

$$
\frac{\partial u}{\partial x}+\frac{\partial v}{\partial y}=0
$$

Equation for in conservation of momentum:

$x$-direction

$$
\frac{\partial u}{\partial t}+u \frac{\partial u}{\partial x}+v \frac{\partial u}{\partial y}=-\frac{1}{\rho} \frac{\partial p}{\partial x}+v\left(\frac{\partial^{2} u}{\partial x^{2}}+\frac{\partial^{2} u}{\partial y^{2}}\right)
$$

and in $y$-direction

$$
\frac{\partial v}{\partial t}+u \frac{\partial v}{\partial x}+v \frac{\partial v}{\partial y}=-\frac{1}{\rho} \frac{\partial p}{\partial y}+v\left(\frac{\partial^{2} v}{\partial x^{2}}+\frac{\partial^{2} v}{\partial y^{2}}\right)
$$


Let $\mathrm{x}$ be the direction of the flow, $\mathrm{y}$ the direction perpendicular to the flow. Suppose there is no velocity component perpendicular to the direction of the flow. As a result the equation of conservation of mass reduces to $\frac{\partial u}{\partial x}=0$, and this leads to $u=u(y)$. Then the Eq. (2) and Eq.(3) reduce to

$$
\begin{aligned}
& 0=\frac{\partial p}{\partial y} . \\
& \frac{\partial u}{\partial t}=-\frac{1}{\rho} \frac{\partial p}{\partial x}+v \frac{\partial^{2} u}{\partial y^{2}}
\end{aligned}
$$

Let $L, U_{0}, p$ denote the characteristic length, velocity, pressure and $x^{\prime}, y^{\prime}, u^{\prime}, v^{\prime}, p^{\prime}$ be the dimensionless number such that

$$
x^{\prime}=\frac{x}{L}, u^{\prime}=\frac{u}{U_{0}}, p^{\prime}=\frac{p}{\rho U_{0}{ }^{2}}, t^{\prime}=\frac{U_{0}}{L} t .
$$

Then the Eq.(4.a)and Eq. (4.b) reduces to

$$
\begin{aligned}
& \frac{\partial u^{\prime}}{\partial t}=-\frac{\partial p^{\prime}}{\partial x^{\prime}}+\frac{1}{\operatorname{Re}} \frac{\partial^{2} u^{\prime}}{\partial y^{\prime 2}} \\
& 0=\frac{\partial p^{\prime}}{\partial y^{\prime}}
\end{aligned}
$$

where Re represents the Reynolds number and

$$
\operatorname{Re}=\frac{U_{0} h}{v} .
$$

Dropping the superscripts, we have

$$
\begin{aligned}
& \frac{\partial u}{\partial t}=-\frac{\partial p}{\partial x}+\frac{1}{\operatorname{Re}} \frac{\partial^{2} u}{\partial y^{2}} \\
& 0=\frac{\partial p}{\partial y} .
\end{aligned}
$$

\section{Plane Poiseuille Flow:}

Let us consider the unsteady two dimensional flow of incompressible viscous fluid flowing between two fixed parallel plates $y=0$ and $y=1$. Eq. (8) shows that $p$ does not depends on $y$. Hence $p$ is a function of $x$ and t. 
Suppose

$$
-\frac{\partial p}{\partial y}=F(t)
$$

Eq.(7) becomes

$$
\frac{\partial u}{\partial t}=F(t)+\frac{1}{\operatorname{Re}} \frac{\partial^{2} u}{\partial y^{2}}
$$

Boundary conditions:

$$
\begin{aligned}
& u=0 \quad \text { at } y=0 \text { and } u=U(t) \text { at } y=1 \text { and initially when } \\
& t=0, u(y, 0) \\
& =-\frac{\operatorname{Re}}{2} F(0) y^{2}+\left\{U(0)+\frac{\operatorname{Re}}{2} F(0)\right\} y
\end{aligned}
$$

\section{Case I:}

We choose a nonlinear function of time as

$$
F(t)=\alpha+\beta t+t^{2} .
$$

For the above function, we have

$$
\frac{\partial u}{\partial t}=\alpha+\beta t+t^{2}+\frac{1}{\operatorname{Re}} \frac{\partial^{2} u}{\partial y^{2}}
$$

General solution of Eq.(12) is

$$
u=\left(k_{1} \cos \sqrt{\mu} y+k_{2} \sin \sqrt{\mu} y\right) e^{-\frac{\mu t}{R}}+\alpha t+\frac{\beta t^{2}}{2}+\frac{\mu^{3}}{6}
$$

where $k_{1}$ and $k_{2}$ are arbitrary constants.

Boundary conditions:

$$
u=0 \quad \text { at } y=0 \text { and } u=U(t) \text { at } y=1 .
$$

Applying the boundary condition in (13), we have

$$
u=\left(\alpha t+\frac{\beta t^{2}}{2}+\frac{\chi^{3}}{6}\right)\left(1+\frac{\sin y \sqrt{\mu}}{\sin \sqrt{\mu}}-\cos y \sqrt{\mu}\right)+\frac{\sin y \sqrt{\mu}}{\sin \sqrt{\mu}} U(0) e^{-\frac{\mu t}{\mathrm{Re}}}
$$


Velocity profile in this case for Reynolds number 1,3 and 5 is given in the Figure 1.

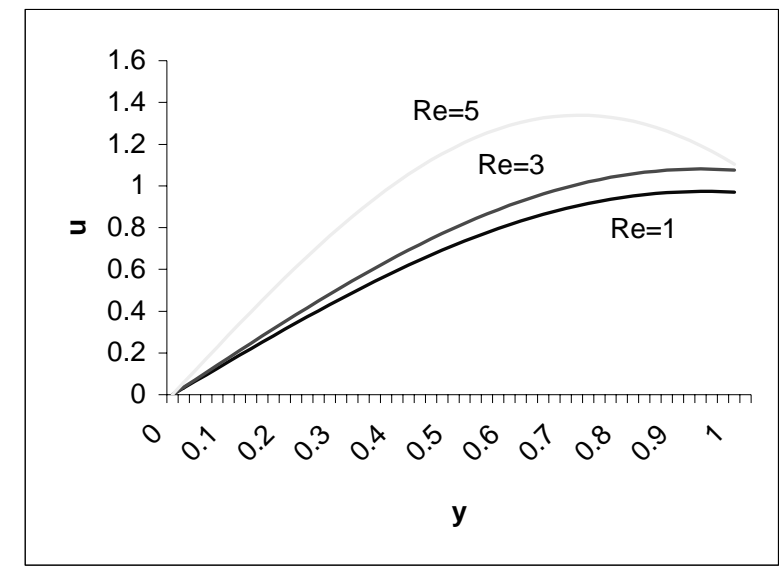

Figure-1

\section{Case II:}

We choose a transient function of time as the following form

$$
F(t)=\alpha e^{-t \beta} \text {. }
$$

For the above function, we have

$$
\frac{\partial u}{\partial t}=\alpha e^{-t \beta}+\frac{1}{\operatorname{Re}} \frac{\partial^{2} u}{\partial y^{2}}
$$

General solution of Eq.(15) is

$$
u=\left(k_{1} \cos \sqrt{\mu} y+k_{2} \sin \sqrt{\mu} y\right) e^{-\frac{\mu t}{\mathrm{Re}}}-\frac{\alpha}{\beta} e^{-t \beta}
$$

where $k_{1}$ and $k_{2}$ are arbitrary constants.

Boundary conditions:

$$
u=0 \quad \text { at } y=0 \text { and at } y=1 .
$$

Applying the boundary condition in Eq. (16), we have the solution

$$
\begin{aligned}
& u=\frac{\alpha}{\beta} \cos \sqrt{\mu} y e^{-t \beta}+\left[-\frac{\mathrm{Re}}{2} \alpha y^{2}+\left\{U(0)+\frac{\operatorname{Re} \alpha}{2}\right\} y+\frac{\alpha}{\beta}(1-\cos \sqrt{\mu} y)\right] e^{-\frac{\mu t}{\mathrm{Re}}} \\
& -\frac{\alpha}{\beta} e^{-t \beta} .
\end{aligned}
$$


Velocity profile in this case for Reynolds number 1,3,5 is given in the Figure 2.

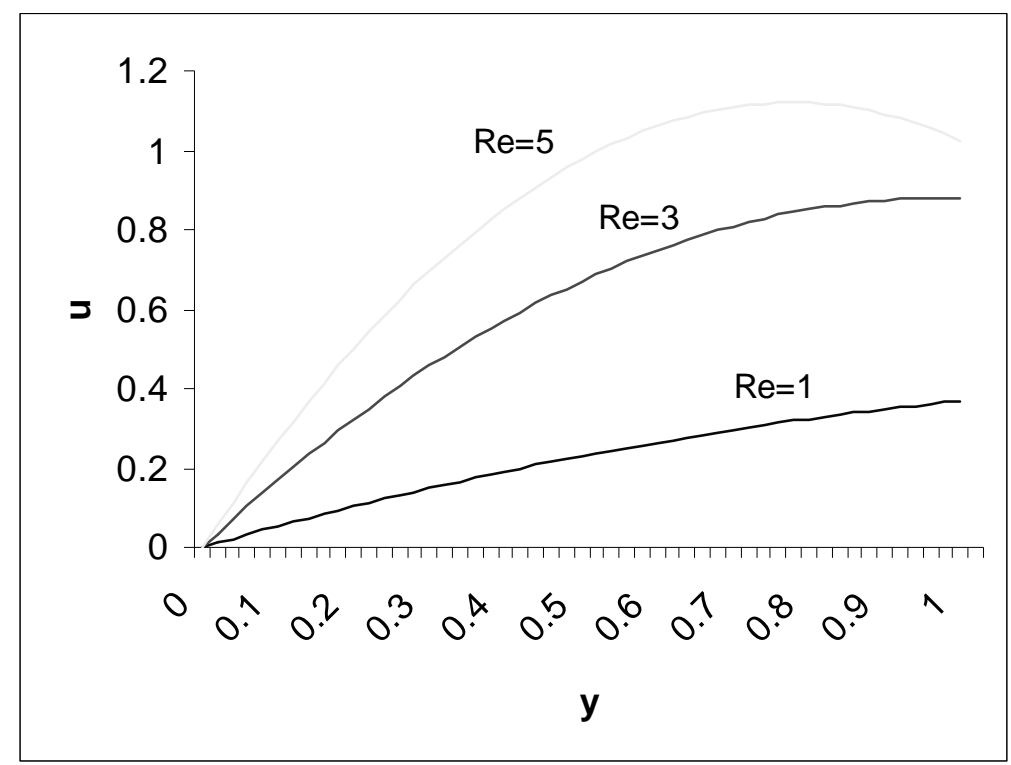

Figure-2

\section{Case III:}

We choose a function for which the pressure gradient varies as a periodic function of time, i. e.

$$
F(t)=\alpha \cos t \beta
$$

For the above function, we have

$$
\frac{\partial u}{\partial t}=\alpha \cos t \beta+\frac{1}{\operatorname{Re}} \frac{\partial^{2} u}{\partial y^{2}} .
$$

General solution of the Eq.(18) is

$$
u=\left(k_{1} \cos \sqrt{\mu} y+k_{2} \sin \sqrt{\mu} y\right) e^{-\frac{\mu t}{\mathrm{Re}}}+\frac{\alpha}{\beta} \sin t \beta
$$

where $k_{1}$ and $k_{2}$ are arbitrary constants.

Applying the boundary condition in Eq.(19), we have

$$
u=\left[-\frac{\operatorname{Re}}{2} \alpha y^{2}+\left\{U(0)+\frac{\operatorname{Re} \alpha}{2}\right\} y\right] e^{-\frac{\mu t}{\operatorname{Re}}}+\frac{\alpha}{\beta} \sin t \beta .
$$


Velocity profile in this case for Reynolds number 1, 3, 5 is given by the Fig-3.

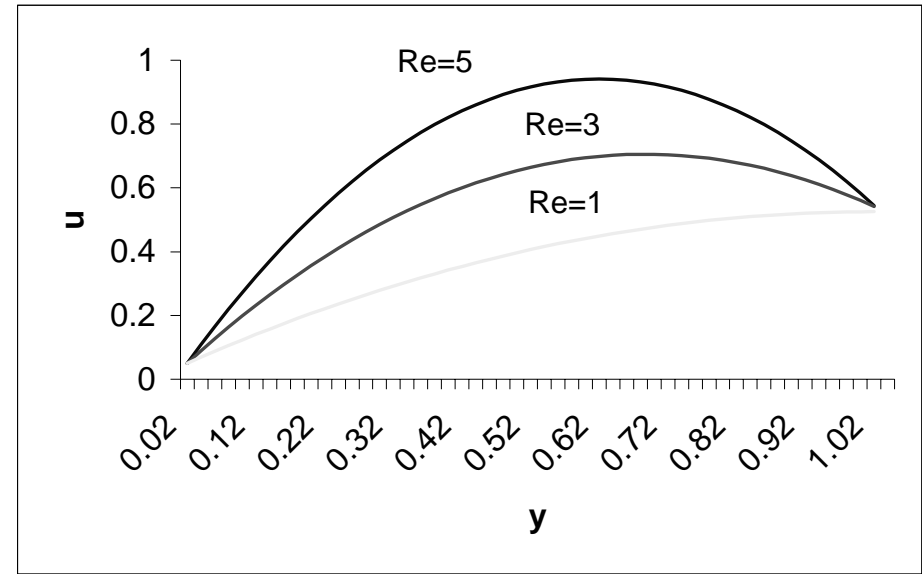

Figure-3

Plane Couette flow: $F(t)=0$

Let us consider the unsteady two-dimensional flow of incompressible viscous fluid between two parallel plates. Suppose one plate $y=0$ is fixed and the other plate is moving with speed U. Again suppose the distance between the plates is one unit. In this case the differential equation for the flow will be

$$
\frac{\partial u}{\partial t}=\frac{1}{\operatorname{Re}} \frac{\partial^{2} u}{\partial y^{2}} .
$$

To solve the above equation we consider the following three cases:

Case I:

$$
U(t)=u_{0}+u_{1} t
$$

General solution of Eq.(21) is

$$
u=\left(k_{1} \cos \sqrt{\mu} y+k_{2} \sin \sqrt{\mu} y\right) e^{-\frac{\mu t}{\operatorname{Re}}}
$$

where $k_{1}$ and $k_{2}$ are arbitrary constants.

Boundary conditions:

$$
u=0 \text { at } y=0 \text { and } u=u_{0}+u_{1} t \text { at } y=1 \text { and } u(0)=u_{0} y .
$$

Applying the above boundary condition in Eq. (22), we have

$$
\left.u=\left(u_{0}+t u_{1}\right)\right) \frac{\sin \sqrt{\mu} y}{\sin \sqrt{\mu}} .
$$


Velocity profile in this case is given by the Fig-4.

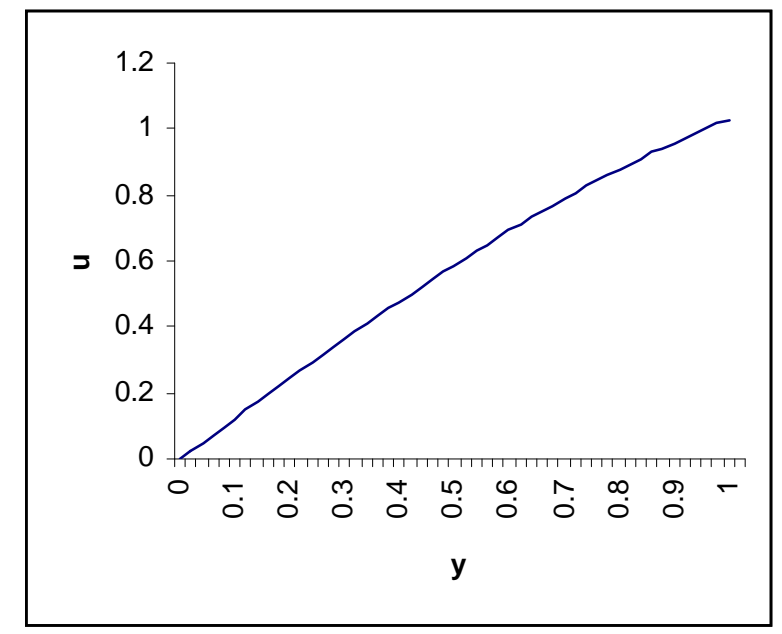

Figure-4

\section{Case II:}

$$
U(t)=u_{0} e^{t u_{1}}
$$

Boundary conditions:

$$
u=0 \text { at } y=0 \text { and } u=u_{0} e^{t u_{1}} \text { at } y=1 \text { and } u(0)=u_{0} y .
$$

Applying the above boundary conditions, the solution of Eq.(21) is

$$
u=\frac{u_{0} \sin \sqrt{\mu} y}{\sin \sqrt{\mu}} e^{t u_{1}} .
$$

Velocity profile in this case is given by the Fig-5.

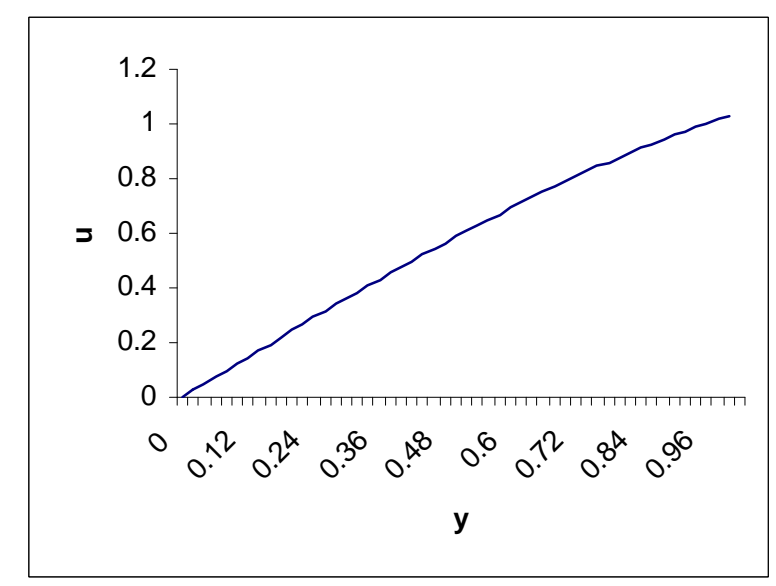

Figure-5 


\section{Case III:}

$$
U(t)=u_{0} \cos u_{1} t .
$$

Applying the boundary conditions of case II the solution of Eq. (21) is

$$
u=\left(u_{0} \sin \sqrt{\mu} y\right) \frac{\cos u_{1} t}{\sin \sqrt{\mu}} .
$$

Velocity profile in this case is given by the Fig-6.

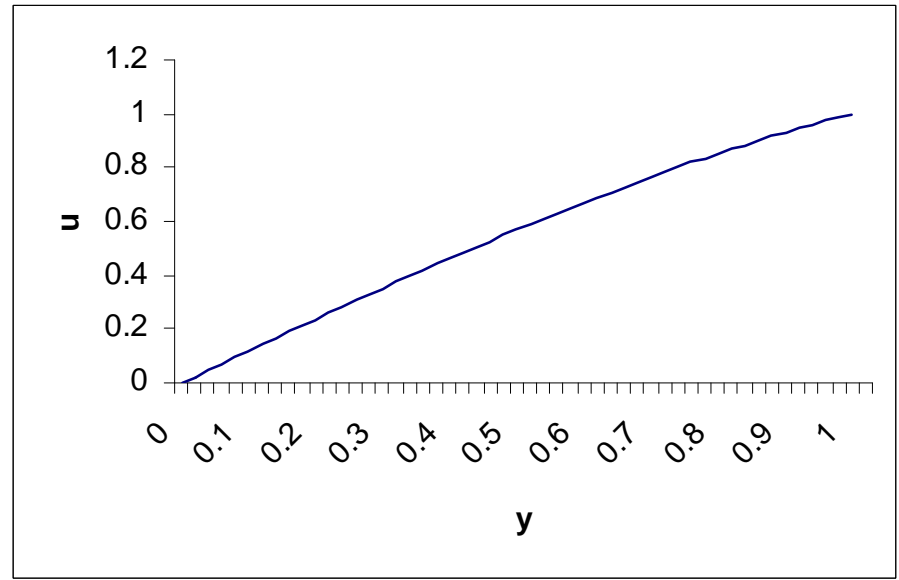

Figure-6

Special case: $\operatorname{Re}=1$

Boundary conditions:

$u=\sin \pi x$ at $t=0$ for $0 \leq x \leq 1$ and $u=0$ at $x=0$ and $x=1$ for $\mathrm{t}>0$.

To solve this problem by finite difference method, we let

$$
\begin{aligned}
& y=i h, i=0,1,2,3, \ldots \ldots \ldots \ldots \ldots \ldots \ldots . . . \\
& t=j k, j=0,1,2,3, \ldots \ldots \ldots \ldots \ldots \ldots \ldots . .
\end{aligned}
$$

Setting $r=\frac{k}{h^{2}}$ and using Crank-Nicolson method, the solution of the problem is given by

$$
u_{i}=\frac{r}{2(1+r)}\left(u_{i-1}+u_{i+1}\right)+\frac{c_{i}}{(r+1)}
$$

where

$$
c_{i}=u_{i, j}+\frac{1}{2} r\left(u_{i-1, j}-2 u_{i, j}+u_{i+1, j}\right) .
$$


Finally, using Jacobi's iteration formula we have the following velocity profile given by Fig 7.

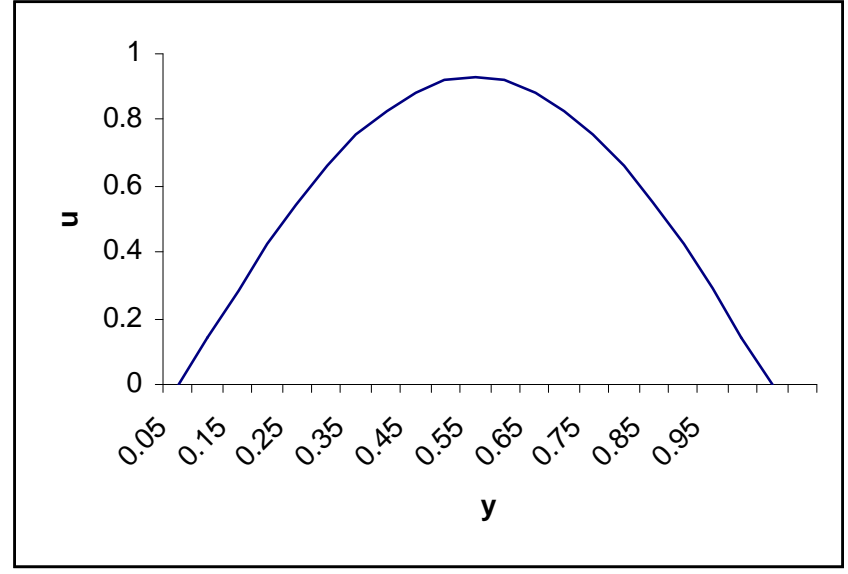

Figure-7

\section{Results and discussion}

It is clear from the Fig-1, Fig-2, Fig-3 and Fig-7 that the velocity profiles are very similar to the parabolic nature of the flow, firstly starts with zero velocity and then gradually increases and attain a maximum velocity. From Fig- 1 to Fig-3 it is clear that the velocity profiles depend on the Reynolds number and the velocity increases with the increasing value of Reynolds number.

\section{REFERENCES}

1. Batchelor G.K.: An introduction to fluid dynamics, Cambridge University Press (1967).

2. Chorlton F.: The Text Book of Fluid Dynamics, English Language Book Society and Vam Nostrand Reinhold Company, London, (1970).

3. Ghose S.K. \& Sengupta P.R.: J. Sci. Res. Vol. 10, No.23, (1988), 117-122.

4. Lamb H.: Hydrodynamics, Dover Publication, New York (1945).

5. Landau L.D. \& Lifshitz E.M.: Fluid Mechanics, Pergaman Press, Oxford, New York, (1989).

6. Milne Thomson L.M.: Theoretical Hydrodynamics, New York, The Mac Millon Company, ((1995).

7. Pai S. I.: Viscous flow theory -Laminas flow, Princeton N. J. D. Van Nostrand Co. INC.(1956).

8. Sengupta P. R.: Some Aspects of Mechanics of Continua Presidential Address, Section of Mathematics, India Science Congress Association Calcutta, (1994-95).

9. Sengupta P.R., Rahman B \& Kandar D K, Unsteady viscous flow between two parallel flat plates, Proc. of Math. Soc. B.H.U. Vols. 15 \& 16 (1999, 2000). 